\title{
A VERSATILE COLUMN LAYOUT FOR THE LANSCE UPGRADE.*
}

\author{
R. Keller, J. M. Verbeke, P. Scott, M. Wilcox, L. Wu, and N. Zahir \\ E. O. Lawrence Berkeley National Laboratory, Berkeley, CA
}

\begin{abstract}
An upgrade program for the LANSCE facility at Los Alamos National Laboratory [1] aims at generating and transporting a $40-\mathrm{mA} \mathrm{H}^{-}$beam with $80 \mathrm{keV}$ energy and minimal emittance increase over the value currently measured with a 16-mA beam. Within a collaboration between the two laboratories, LBNL has built a new ion source [2] that allows the extraction of a $40-\mathrm{mA}$ beam. However, there are strong indications that a new layout of the extraction system/LEBT is needed to efficiently deliver a low-emittance beam with this increased beam current. An additional requirement for the new system is its adaptability to a range of beam currents between 20 and 40 $\mathrm{mA}$. The design of this new system, based on extensive simulations with the code IGUN [3], is described in this paper.
\end{abstract}

\section{INTRODUCTION}

The LANSCE facility at Los Alamos National Laboratory is currently operating with a $16-\mathrm{mA} \mathrm{H}^{-}$ion beam obtained from a surface-production ion source [4]. A project to improve the neutron output of the facility is now underway and aims at an ion-beam current-increase of a factor of two. Beam simulations [1], however, clearly indicate that the available $80-\mathrm{kV}$ column that incorporates the extraction system and the initial, electrostatic, part of the LEBT will be unable to transport beams of this intensity through the subsequent magnetic LEBT.

Members of the Ion Beam Technology Program at Berkeley Lab were already engaged in providing a $40-\mathrm{mA}$ ion source [2] for the LANSCE upgrade project, and the work presented in this paper was undertaken to develop a viable modification to the existing $80-\mathrm{kV}$ column. One main constraint for this work was the condition that the existing insulators be preserved in order to keep this project within budget and schedule. Another condition was imposed by the magnetic LEBT whose first solenoid aperture of $38-\mathrm{mm}$ diameter was not to be illuminated to more than $50 \%$.

\section{BASIC CONSTRAINTS}

\subsection{0-kV Column}

The existing 80-kV column in the LANSCE injector re-

*Work supported by the Director, Office of Science, Office of Basic Energy Sciences, of the US Department of Energy under Contr. No. DE-AC03-76SF00098. presents a modified Pierce extraction system [5] with two intermediate electrodes between source outlet potential and ground. The electrode contours are shaped according to Pierce's design, but the voltage in the first gap had to be considerably reduced to avoid frequent high-voltage breakdown. In addition, the aperture in the first intermediate electrode was widened from 10 to $22 \mathrm{~mm}$ which moved the column parameters even further away from a true Pierce system. A trap is added on the downstream side of the column electrodes to keep space-charge neutralizing, positive, ions within the transported $\mathrm{H}^{-}$ beam.

The column insulator is shown in Fig. 1, already fitted with the new electrodes that will be discussed later on. It consists of two concentric ceramic tubes enclosing a space that can be filled with an insulating gas. With this construction, high-voltage breakdown in air is totally avoided up to a total voltage of $80 \mathrm{kV}$. The inner tube carries corona rings on its outer perimeter which are connected to feed-throughs that pass through this insulator and connect to the inner electrodes. Small recesses on the inner perimeter of this tube serve as indices for the installed electrodes.

\subsection{Ion Source}

Both, the old and the new ion source are of the surfaceproduction type with internal, cesium-covered converter [2]. The converter bias of $-250 \mathrm{~V}$ with respect to the outlet-electrode potential already accelerates the $\mathrm{H}^{-}$ion beam within the plasma generator and helps to overcome problems arising from high space-charge density at thermal ion velocities usually encountered with other ion sources. An arrangement of permanent magnets inside the plasma electrode significantly reduces the amount of electrons that are extracted together with the $\mathrm{H}^{-}$ions.

\subsection{IGUN simulations}

The beam-trajectory simulation code IGUN [3] is capable of handling positive ions extracted from a plasma or "injected" into a system under study as an external input file. Our rationale in using IGUN as a design tool for the LANSCE column upgrade was that we did not intend to study the fundamental processes of $\mathrm{H}^{-}$beam formation but rather build on an existing system and investigate the effects caused by the higher space-charge density of the envisaged 40-mA beam. For this purpose, assuming a proton beam with inverted electrode potentials was judged to be adequate. 


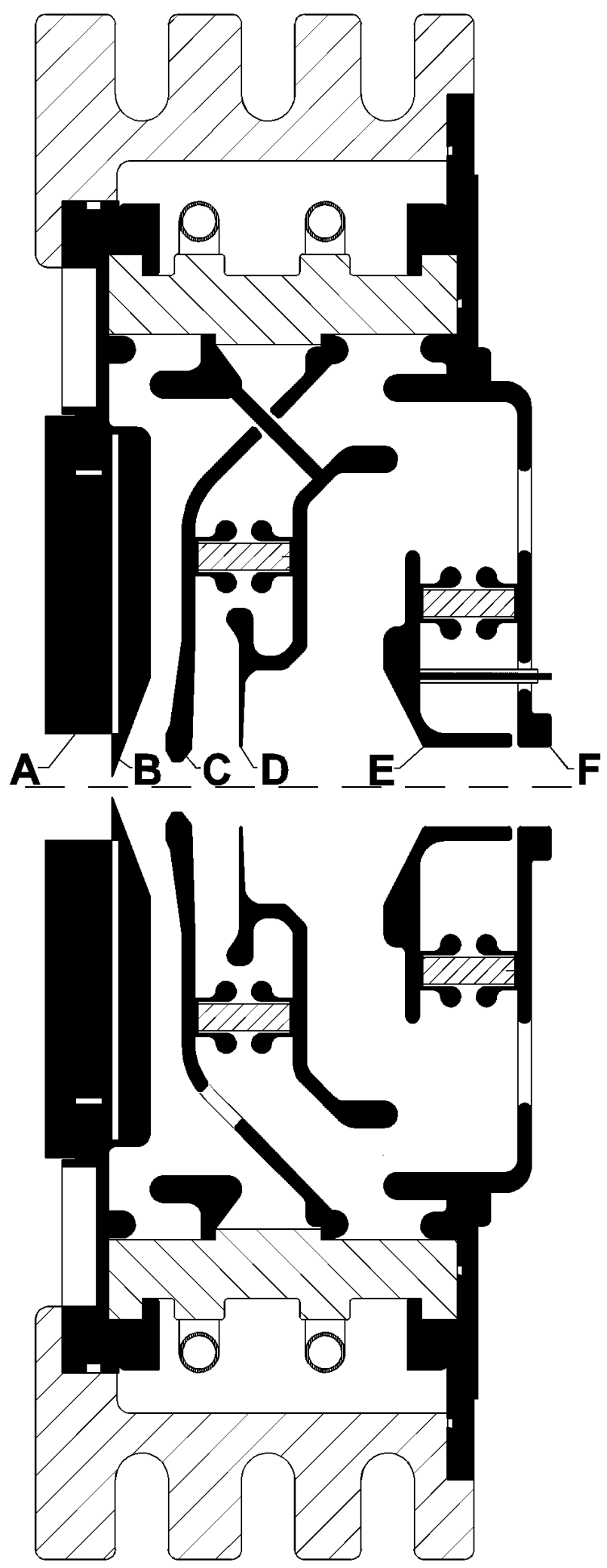

Figure 1. New 80-kV column for the LANSCE injector. $\mathrm{A}$, ion-source plasma-electrode. B, outlet ("Pierce") electrode on $-80 \mathrm{kV}$. C, extractor electrode $(-50 \mathrm{kV})$. D, lens $(-75 \mathrm{kV})$. E, ion trap $(+8 \mathrm{kV})$. F, ground electrode. All potentials apply to negative ions.
The simulations had the goal of creating an electrostatic LEBT as first part of the existing hybrid LEBT capable of accommodating a wide range of beam currents, between 15 and $40 \mathrm{~mA}$, and with minimized aberrations to keep the effective beam emittance as low as possible.

In most of the simulations performed for this study, a longitudinal energy of $375 \mathrm{eV}$ was assigned to the ions within the ion-source plasma. The exact value of this energy, down to $250 \mathrm{eV}$, proved to have only a weak influence on the resulting beam parameters. Because of the fact that reduction of aberrations was a primary goal in this study, most of the calculations were performed for vanishing ion temperature, but for emittance evaluations, ion temperatures of $1 \mathrm{eV}$ were assumed.

As stated above, the existing LEBT relies on spacecharge compensation for the ion beam drifting between its electrostatic (column) and magnetic parts. To simplify our simulation work and significantly cut down on the CPU times, we did not calculate complete beam trajectories over the full drift length but confined the range of calculations to the column itself, with the implicit assumption that the ion trajectories remain straight after passing the first equipotential on slightly negative potential from the downstream side (the sign convention here applies to a positive ion beam).

To adequately judge the simulation results, however, trajectories were traced to the entrance plane of the first magnetic solenoid lens, $300 \mathrm{~mm}$ downstream of the column entrance plane. Up to $19 \mathrm{~mm}$ beam radius in this plane was regarded as acceptable. Once we arrived at a viable design we split the simulation problem into two parts, performing the calculations near the outlet electrode at twice the resolution applied to the remaining part.

\section{NEW COLUMN DESIGN}

As a first approach to developing the new column, we pursued the idea of a "compound system" [6] which consists of a low-voltage extraction gap followed by an electrostatic einzel lens and the main gap that determines the final beam energy. We soon realized, however, that such a system would occupy more longitudinal space than the existing insulator could accommodate and therefore decided to integrate two of the functions, i. e. those of lens and main gap, into one element which is labeled as 'D' in Fig. 1.

Another condition we imposed was to keep the extractor electrode, $\mathrm{C}$, at least as far away from the outlet electrode and the system axis as the first intermediate electrode of the existing column has been, ensuring that the risk of high-voltage breakdown caused by stray particles coming from the source is not increased in the new design. We did, however, raise the voltage in the gap between the outlet and first intermediate electrode, B and $\mathrm{C}$, because we felt this voltage had been chosen with extreme conservatism and did not by itself actually contribute to sparking. 


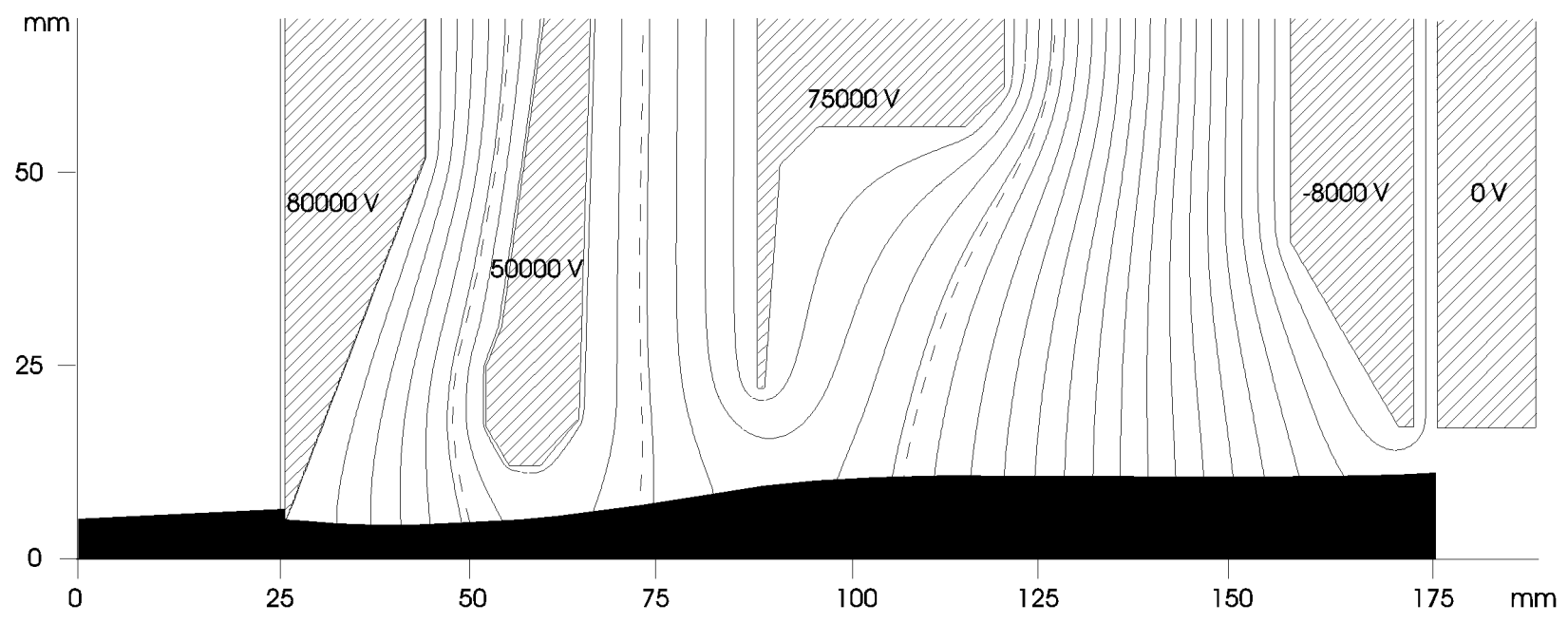

Fig. 2. Simulated beam in the optimized electrode configuration. Electrode potentials as marked in this figure apply to a positive ion beam. The contour of the ground electrode on the right has been graphically added to the contours actually used in the IGUN runs. The second dashed equipotential line, between the 50- and 75-kV electrodes, marks the transition surface for split calculations as described in the text.

After we gained some experience with the intricacies of this design we substantially modified the contour of the lens, D, from a cylinder to the bell shape shown in Fig. 1. We also shortened the length of the ion trap electrode, E, because it contributes to the path length the ion beam has to traverse under the influence of its full space charge, increasing its radial growth. This change is not shown in Fig. 1, but rather in the integral trajectory plot in Fig. 2.

\section{RESULTS}

Emittance plots for the first and second parts of the optimized configuration are given in Fig. 3. The first gap of the new column is virtually aberration-free, whereas the main gap produces a faint distortion, only visible in the zerotemperature condition.

After the new electrodes were assembled within the insulator the system could be immediately conditioned to the design voltage of $80 \mathrm{kV}$ [7].

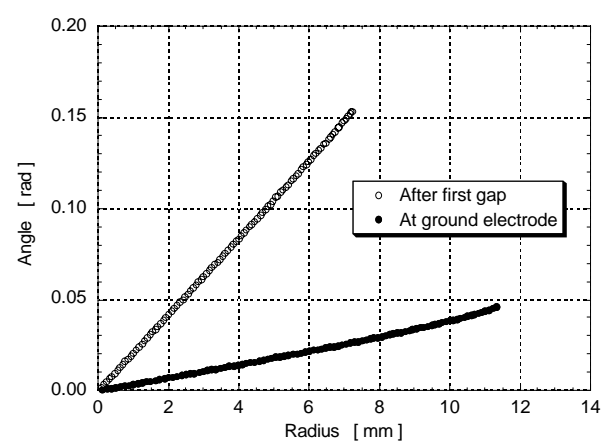

Fig. 3. Emittance plots for the optimized configuration.

\section{ACKNOWLEDGMENTS}

The authors thankfully acknowledge the contributions by K.-N. Leung, Berkeley Lab, who initiated this collaboration with the LANSCE group and by R. R. Stevens Jr., Los Alamos Nat. Lab., for frequent exchange of technical information and suggestions how to improve the column design. The mechanical design of the new column configuration was carried out by C. Matuk, Berkeley Lab.

\section{REFERENCES}

[1] R. R. Stevens, W. Ingalls, O. Sander, B. Prichard, and J. Sherman, "Beam Simulations for the H- Upgrade at LANSCE," Proc. 1998 Linac Conf., Chicago, IL.

[2] M. Williams, R. Gough, R. Keller, K. Leung, D. Meyer, A. Wengrow, O. Sander, W. Ingalls, B. Prichard, and R. Stevens, "Ion Source Development for LANSCE Upgrade," Proc. 1998 Linac Conf., Chicago, IL.

[3] R. Becker, "New Features in the Simulation of Ion Extraction with IGUN," Proc. EPAC 98, Stockholm, Sweden (1998)

[4] R. L. York and Ralph R. Stevens, Jr., "Third International Symposium on Production and Neutralization of Negative Ions and Beams," Brookhaven National Laboratory, 1983, p. 410.

[5] J. R. Pierce, "Theory and Design of Electron Beams," Van Nostrand, Toronto, 1954, pp. 177 - 181.

[6] R. Keller, "Ion Extraction," in I.G. Brown, ed., "The Physics and Technology of Ion Sources," John Wiley, New York, 1989.

[7] R. R. Stevens, Jr., private communication (1999). 